\title{
$N$-methylphenylalanyl-dehydrobutyrine diketopiperazine, an A-factor mimic that restores antibiotic biosynthesis and morphogenesis in Streptomyces globisporus 1912-B2 and Streptomyces griseus 1439
}

\author{
Bohdan Matselyukh ${ }^{1}$, Fatemeh Mohammadipanah ${ }^{2}$, Hartmut Laatsch ${ }^{2}$, Jürgen Rohr ${ }^{3}$, Olga Efremenkova ${ }^{4}$ and \\ Volodymyr Khilya ${ }^{5}$
}

The cell-free extracts of a landomycin E-producing strain, Streptomyces globisporus 1912-2, were shown to contain a lowmolecular-weight compound that, like $A$-factor, restored the landomycin $\mathrm{E}$ and streptomycin biosynthesis and sporulation of the defective mutants S. globisporus 1912-B2 and S. griseus 1439, respectively. The compound was purified by thin layer chromatography and HPLC. It had an absorption maximum at $\lambda_{\max }=245 \mathrm{~nm}$ and a molecular mass of $\mathrm{m} / \mathrm{z} 244$. On the basis of NMR spectroscopy $\left({ }^{1} \mathrm{H},{ }^{13} \mathrm{C}, \mathrm{HSQC}, \mathrm{HMBC}, \mathrm{COSY}\right.$ and NOE) the chemical structure of the compound was elucidated as 6-benzyl-3-eth-(Z)-ylidene-1-methyl-piperazine-2,6-dione ((L)- $N$-methylphenylalanyl-dehydrobutyrine diketopiperazine (MDD)). The sequences of arpA genes in S. globisporus 1912-2 and S. griseus NBRC 13350 are highly conserved. An explanation for the observed biological activity of MDD was proposed.

The Journal of Antibiotics (2015) 68, 9-14; doi:10.1038/ja.2014.86; published online 9 July 2014

\section{INTRODUCTION}

Communication systems (quorum sensing) among Gram-positive and Gram-negative bacteria involve diffusible signal molecules $(\gamma$ butyrolactones, $\mathrm{N}$-acyl homoserine lactones, diketopiperazines (DKPs), furans and other molecules) interacting with specific cell surface-associated or intracellular receptors, thereby inducing genes that control biofilm formation, production of antibiotics and virulence factors, morphogenesis of cells and other functions. ${ }^{1-5}$

$A$-factor-dependent cascade regulation is one of the important systems of secondary metabolism and morphogenesis regulation in streptomycetes. 6,7 The $A$-factor belongs to the family of $\gamma$-butyrolactones, small signaling molecules that establish communication between the neighboring hyphae of streptomycetes in nanomolar concentrations. ${ }^{3,8}$ It binds to the receptor protein ArpA, causing its dissociation and consequently activation of transcription of the genes of the AdpA regulon, which control aerial mycelium formation as well as the biosynthesis of antibiotics and pigments. The protein AdpA binds to 37 regions of the DNA, activating 72 genes of streptomycetes, many of whose functions have not yet been assigned. ${ }^{9}$ A variety of $\gamma$-butyrolactones from streptomycetes with different chemical structures is known. Each receptor protein in various Streptomyces species is highly specific for its cognate $\gamma$-butyrolactone. ${ }^{6,10}$

Streptomyces globisporus 1912 produced the red-orange antitumor antibiotic landomycin E, a member of the angucycline family. Landomycin E induces apoptosis in cancer cells, including those resistant to doxorubicin. ${ }^{11}$ The biosynthesis of landomycin $\mathrm{E}$ is encoded by the Ind-cluster, which consists of 27 structural and 3 regulatory genes. ${ }^{12}$ The wild-type strain S. globisporus 1912 and many of its mutants, including mutant 1912-2, produce compounds, with $R_{f} 0.4$ and 0.8 , respectively, which were found to be able to restore the biosynthesis of landomycin $\mathrm{E}$ and sporulation of the antibiotically inactive mutant 1912-B2. ${ }^{13} \mathrm{~A}$ preliminary description of the compound with $R_{f} 0.4$ and its relation to the compound with $R_{f} 0.8$ was published earlier. ${ }^{14}$ The subject of the present paper was the structure elucidation of the compound with $R_{f} 0.4$ as a new DKP, and also description of its biochemical and biological properties.

\footnotetext{
${ }^{1}$ Department of Genetics of Microorganisms, D.K. Zabolotny Institute of Microbiology and Virology of the National Academy of Sciences of Ukraine, Kyiv, Ukraine; ${ }^{2}$ Department of Chemistry, Institute for Organic and Biomolecular Chemistry, University of Göttingen, Göttingen, Germany; ${ }^{3}$ Department of Pharmaceutical Sciences, College of Pharmacy, University of Kentucky, Lexington, USA; ${ }^{4}$ Sector of Screening of Natural Compounds, G.F. Gause Institute of New Antibiotics, Russian Academy of Medical Sciences, Moscow, Russia and ${ }^{5}$ Department of Organic Chemistry, Taras Shevchenko National University of Kyiv, Kyiv, Ukraine

Correspondence: Professor B Matselyukh, Department of Genetics of Microorganisms, D.K. Zabolotny Institute of Microbiology and Virology of the National Academy of Sciences of Ukraine, Zabolotnoho Street 154, Kyiv D 03680, Ukraine.

E-mail: bohdan.matselyukh@gmail.com

Received 22 February 2014; revised 13 May 2014; accepted 22 May 2014; published online 9 July 2014
} 


\section{MATERIALS AND METHODS}

\section{Strains and media}

The objects of the investigation were the sporulating strains S. globisporus 1912-2 and S. griseus 773, the producers of landomycin E and streptomycin, respectively, and their antibiotically inactive mutants S. globisporus 1912-B2 and S. griseus 1439, defective in biosynthesis of the (L)-N-methylphenylalanyldehydrobutyrine diketopiperazine (MDD) and the $A$-factor, respectively. ${ }^{13}$ The $A$-factor-deficient strain 1439 was obtained by nitrosomethylbiuret treatment of S. griseus 32-12. ${ }^{15}$ The streptomycin-sensitive and -resistant strains of S. levoris 165 were used for the detection of streptomycin production. The streptomycetes were grown on corn-soy medium consisting of corn meal $20 \mathrm{~g}$, soy meal $10 \mathrm{~g}, \mathrm{NaCl} 5 \mathrm{~g}$, agar $10 \mathrm{~g}$ in 11 distilled water, $\mathrm{pH} 7$, with sterilization at 1 bar overpressure for $30 \mathrm{~min}$. Cultures of the strains 773 and 1912-2 produced the $A$-factor and MDD during 5-6 days of growth on minimal solid medium at $28^{\circ} \mathrm{C}$, containing asparagine $1 \mathrm{~g}$, glycine $1 \mathrm{~g}, \mathrm{~K}_{2} \mathrm{HPO}_{4} 0.5 \mathrm{~g}, \mathrm{MgSO}_{4}$ $0.2 \mathrm{~g}, \mathrm{NaCl} 4 \mathrm{~g}$, glycerol $15 \mathrm{~g}$, agar $10 \mathrm{~g}$, trace elements $\left(\mathrm{FeSO}_{4}, \mathrm{CuCl}_{2}, \mathrm{MnSO}_{4}\right.$, $\mathrm{CaCl}_{2}, 10.0 \mathrm{mg}$ each) in 1.01 distilled water, $\mathrm{pH} 7.2$, with sterilization at 0.75 bar overpressure for $30 \mathrm{~min}$. The same solid medium in ten Petri dishes was used for examination of production of the MDD and A-factor by the 1912-B2 and 1439 strains, respectively, after adding the corresponding cross-regulators ( $1 \mu \mathrm{g}$ of the MDD or $100 \mathrm{ng}$ of the $A$-factor in $20 \mathrm{ml}$ of the medium) and cultures growing for 6 days.

\section{Purification of the $A$-factor and MDD}

The minimal solid medium of the grown cultures was cut into cubic pieces and extracted with chloroform-acetone (2:1) to obtain the $A$-factor $((2 S)$-isocapryloyl-(3R)-oxymethyl- $\gamma$-butyrolactone) from strain 773 and the so far unknown inducer MDD of landomycin E biosynthesis, respectively, from strain 1912-2.

The crude extracts were evaporated to dryness using a rotary evaporator and the residues were dissolved in ethanol. The regulators were separated by thin layer chromatography on silica gel 60 F254 (Merck, Darmstadt, Germany) sheets with benzene-ethyl acetate-acetone-ethanol (4:2:1:0.5). The UV-absorbing zones were triturated with ethanol, and the silica gel was removed by centrifugation at 10000 r.p.m. The compound MDD was further purified by HPLC using an HPLC/MS (Agilent Technologies, Waldbronn, Germany) system with UV detector and a Zorbax Hypersyl ODS reversed phase column. This way, $0.5 \mathrm{mg}$ of $A$-factor and $5.0 \mathrm{mg}$ of the MDD were obtained from 5.01 of the corresponding agar culture. The output of $A$-factor corresponds to similar results obtained earlier by other authors. ${ }^{16}$ For comparison, the $A$ factor was also chemically synthesized in the Institute of Bioorganic Chemistry of the Russian Academy of Sciences. Concentrations of the A-factor and of MDD were determined by HPLC.

\section{Biological activity}

The biological activity of the purified $A$-factor and of MDD was examined using test strains 1439 and 1912-B2. Strain 1439 was inoculated on Petri dishes with solid corn-soy or minimal medium as a central stripe, and small paper discs with the $A$-factor $(100.0 \mathrm{ng})$ and $\mathrm{MDD}(1.0 \mu \mathrm{g})$, respectively, were put near the ends of the last. Strain 1912-B2 was inoculated evenly on a solid medium using a glass spatula, and paper discs with the same concentration of the $A$-factor and MDD, respectively, were placed on the surface. The results of the action of both factors on antibiotic biosynthesis and morphogenesis were registered on the second and fifth day of incubation of the strains 1912-B2 and 1439 , respectively. The purified MDD and the chemically synthesized $A$-factor were also added to fresh lawns of 1439 and 1912-B2 solid cultures in 10 Petri dishes $(1.0 \mu \mathrm{g}$ per $20 \mathrm{ml}$ and $100.0 \mathrm{ng}$ per $20 \mathrm{ml}$, respectively). After 6 days of incubation at $28^{\circ} \mathrm{C}$ the agar cultures were extracted using a mixture of chloroform and acetone (2:1), and the resulting extracts were analyzed by TLC. Identification of MDD and the $A$-factor from spots detected under UV light on TLC aluminum sheets was performed by spectrophotometry and HPLC.

Absorption and NMR spectra. NMR spectra of MDD were measured at $125.707 \mathrm{MHz}\left({ }^{13} \mathrm{C}\right.$ NMR) and $300.141 \mathrm{MHz}\left({ }^{1} \mathrm{H}\right.$ NMR) on Varian INOVA 500 and Mercury 300 spectrometers, respectively. The absorption spectra of the ethanol solutions of the A-factor and MDD were recorded on a Beckman
DU-8 spectrophotometer. LC/MS was performed using a liquid chromatograph, Agilent Technologies 1200, with a single quadrupole detector, model G19, and a G1978A ion source in ESI mode was used. The separations were performed on a Zorbax Hypersyl ODS column $\left(12.0 \times 125.0 \mathrm{~mm}^{2}, 3 \mu \mathrm{m}\right)$ with hexane:ethanol (98:2) at a flow rate of $0.17 \mathrm{ml} \mathrm{min}^{-1}$, and with dual detection at $210-400 \mathrm{~nm}$. The chromatograms were analyzed with the Chemstation software.

Configuration. To determine the absolute configuration of the NMePhe unit, $0.4 \mathrm{mg}$ of MDD was hydrolyzed by heating in $0.2 \mathrm{ml} 6 \mathrm{~N} \mathrm{HCl}$ for $24 \mathrm{~h}$ at $110^{\circ} \mathrm{C}$. After cooling, the solution was evaporated to dryness and the residue redissolved in $\mathrm{H}_{2} \mathrm{O}(50 \mu \mathrm{l})$. To the resulting hydrolysate solution or to a solution of the reference amino acids (L-NMePhe and D-NMePhe, respectively, $50 \mu \mathrm{l}$; $50 \mathrm{~mm}$ each), a solution of FDAA (Marfey's reagent, $\mathrm{N}$-(2,4-dinitro-5fluorophenyl)-L-alanineamide, $100 \mu \mathrm{l}$ of $1 \%(\mathrm{w} / \mathrm{v})$ solution in acetone) was added. After addition of $\mathrm{NaHCO}_{3}$ solution $(20 \mu \mathrm{l} ; 1 \mathrm{M})$, the mixture was incubated for $1 \mathrm{~h}$ at $40^{\circ} \mathrm{C}$. The reaction was stopped by addition of $\mathrm{HCl}(10 \mu \mathrm{l}$; $2 \mathrm{M}$ ), the solution was evaporated to dryness, and the residue was re-dissolved in acetonitrile $(1 \mathrm{ml})$. An aliquot of this solution $(20 \mu \mathrm{l})$ was analyzed by HPLC (Phenomenex Luna C18 (National Analytical Corporation, Maharashtra, India), $2504.6 \mathrm{~mm}, 5 \mu \mathrm{m}$; solvents: $\mathrm{A}, \mathrm{H}_{2} \mathrm{O}+0.05 \% \mathrm{HCOOH} ; \mathrm{B}$, $\mathrm{MeOH}+0.05 \% \mathrm{HCOOH}$; linear gradient from $10 \% \mathrm{~B}$ in A at $t=0$ min to $90 \% \mathrm{~B}$ in A within $20 \mathrm{~min} ; 25^{\circ} \mathrm{C} ; 300 \mu \mathrm{lmin}-1$ ). The configuration of $\mathrm{NMePhe}$ was determined by comparing the chromatograms with derivatives of commercially available D- and L- $N$-methyl phenylalanine. The FDAA derivative obtained from the regulator MDD had a retention time of $15.9 \mathrm{~min}$ and thus indicated $\mathrm{L}-\mathrm{N}$-methyl phenylalanine as its chiral component, in comparison with the derivatives of authentic L-NMePhe ( $15.9 \mathrm{~min})$, and DNMePhe (11.6 min).

\section{RESULTS}

Biological activity of the compound with $R_{f} 0.4$ compared to the $A$-factor

Two UV-absorbing compounds were produced by donor strain 19122 (Figure 1a, 1, arrows), which were missing in the mutant recipient strain 1912-B2 (Figure 1a, 2). A labile compound with $R_{f} 0.8$

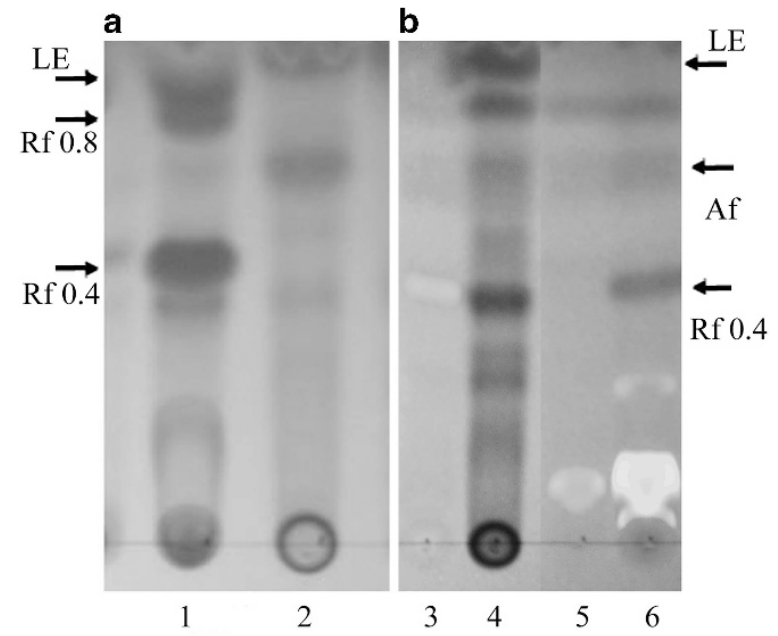

Figure 1 Thin layer chromatogram in UV light of cell-free extracts of the agar cultures: (a) S. globisporus 1912-2 (1) and its inactive mutant 1912B2 (2), grown on minimal medium; the arrows show localization of landomycin $\mathrm{E}$ and new compounds with $R_{f} 0.8$ and $R_{f} 0.4$ (( (L)- $N$ methylphenylalanyl-dehydrobutyrine diketopiperazine (MDD)), respectively. (Reprinted with permission from Matselyukh et al. ${ }^{14}$ (c)2012 National Academy of Sciences of Ukraine). (b) Mutants 1912-B2 $(3,4)$ and S. griseus $1439(5,6)$ grown on minimal medium after addition of $A$-factor (4) and purified MDD (6); the arrows show localization of landomycin E, $A$-factor and MDD. 
a

1439

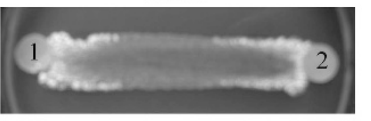

b

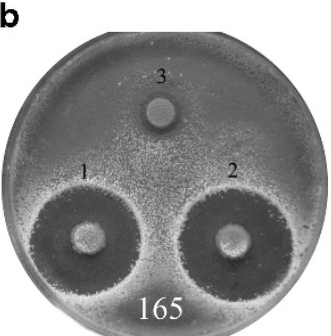

c

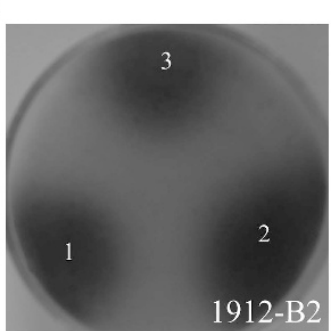

Figure 2 Biological activity of (L)- $N$-methylphenylalanyl-dehydrobutyrine diketopiperazine (MDD): (a) restoration of sporulation of S. griseus 1439 by A-factor (1) and MDD (2); (b) restoration of streptomycin biosynthesis in S. griseus 1439 by A-factor (1) and MDD (2). Agar blocks cut out from sporulating $(1,2)$ and non-sporulating (3) areas of an agar culture of strain 1439 (a) were puted on the lawn of a streptomycin-sensitive culture $S$. levoris 165. Zones of growth inhibition were generated by streptomycin. (c) Restoration of landomycin E biosynthesis in S. globisporus 1912-B2 by A-factor (1), MDD (3) and a compound with $R_{f} 0.8$ (2). (Reprinted with permission from Matselyukh et al. ${ }^{14} \odot 2012$ National Academy of Sciences of Ukraine).

disappeared after 6 days of culture growth and transformed into a stable compound with $R_{f} 0.4$. We then found that the TLC-purified compound with $R_{f} 0.4$, consequently called MDD, restored production of landomycin E and sporulation in a 1912-B2 mutant and displayed a biological activity similar to that of the $A$-factor: MDD restored sporulation (Figure 2a, 1) as well as streptomycin (Figure 2b, 1 ) and $A$-factor production (Figure $1 b, 6)$ in $S$. griseus 1439. For comparison, we also tested an extracted and TLC-purified $A$-factor from a sporulating and streptomycin-producing culture of $S$. griseus 773, as well as a chemically synthesized $A$-factor: Both restored sporulation as well as MDD (Figure 1b,4) and landomycin E (Figure 2c) biosynthesis in S. globisporus 1912-B2. However, the concentration of MDD needs to be one order of magnitude higher than the $A$-factor concentration to reach the above-described biological effects.

\section{Structure elucidation of the new DKP}

HPLC and LC/ESIMS of the purified MDD gave a clear molecular ion signal at $m / z 245(\mathrm{M}+\mathrm{H})^{+}$(Figure 3). ( + )-ESI-HRMS afforded the molecular formula of $\mathrm{C}_{14} \mathrm{H}_{16} \mathrm{~N}_{2} \mathrm{O}_{2}$ (found 267.11036, calcd. 267.11041 for $\left.\mathrm{C}_{14} \mathrm{H}_{16} \mathrm{~N}_{2} \mathrm{O}_{2} \mathrm{Na}\right)$. In the ${ }^{13} \mathrm{C}$ and ${ }^{1} \mathrm{H}$ NMR spectra (Table 1 and Figure 4), respectively, two acid/amide carbonyls were seen, one $\mathrm{OH} / \mathrm{NH}$ signal at low field, diastereotopic methylene protons of an $\mathrm{ABX}$ system, and a conspicuous $\mathrm{CH}-\mathrm{CH}_{3}$ signal with the methine signal at $\delta_{\mathrm{CH}}$ 111.6. A search in AntiBase ${ }^{17}$ delivered a $\mathrm{C}=\mathrm{CH}-\mathrm{CH}_{3}$ fragment as the only plausible substructure. In the $\mathrm{HMBC}$ spectrum, the latter $\mathrm{CH}$ signal gave a cross peak with the carbonyl at $\delta 158.7$, resulting in an $\alpha, \beta$-unsaturated acid/amide (see also Figure 4). A second spin system consisted of a monosubstituted benzene unit, connected with a $\mathrm{CH}_{2}-\mathrm{CH}-\mathrm{CO}$ fragment. The methine proton in this fragment coupled with both carbonyls. A search in AntiBase with these substructures and 13 or 14 carbon atoms resulted in the DKPs cyclo(Phe, dehydroAbu) and cyclo(NMeTyr, dehydroAbu) as the only results. It was clear therefore that the compound is derived from cyclo(Phe, dhydroAbu), where one of the nitrogen atoms is methylated. As the NMe signal and the olefinic methine $\mathrm{H}-3^{\prime}$ coupled with the same carbonyl $\mathrm{Cl}^{\prime}$, both must be neighbors of the latter. Correspondingly, the $\mathrm{NMe}$ singlet gave an HMBC signal with $\mathrm{C}-2$. This resulted in cyclo(NMePhe, dhAbu) $(\mathrm{dhAbu}=$ dehydroaminobutyric acid $)$, which was confirmed by all further correlations (Figure 5). The olefinic methyl gave an NOE signal with the NH singlet and the double bond was therefore believed to have $(Z)$ configuration, which resulted in the structure shown in Figure 5. To determine the absolute configuration of the NMePhe unit, a sample of compound was hydrolyzed and derivatized with
FDAA (Marfey's reagent, $N$-(2,4-dinitro-5-fluorophenyl)-L-alaninamide). The configuration of $\mathrm{NMePhe}$ was determined by comparing the chromatograms with derivatives of commercially available D- and L- $N$-methyl phenylalanine. The FDAA derivative obtained from the compound had a retention time of $15.9 \mathrm{~min}$ and thus indicated L-Nmethyl phenylalanine, by comparison with the derivatives of authentic L-NMePhe $(15.9 \mathrm{~min})$ and $\mathrm{D}-\mathrm{N}-\mathrm{MePhe}(11.6 \mathrm{~min})$. Thereby, the molecular structure of the new compound of antibiotic biosynthesis and morphogenesis in Streptomyces has been elucidated as L-6-benzyl3-eth-(Z)-ylidene-1-methyl-piperazine-2,5-dione (MDD). This compound had been obtained by synthesis previously; however, details on the structure confirmation or the NMR data were not available. ${ }^{18}$

\section{Receptor protein ArpA}

$A$-factor is known to bind to the regulatory domain of the receptor protein ArpA S. griseus NBRC 13350. ${ }^{3}$ Production of ArpA-like protein by $S$. globisporus 1912-2 was not studied. High-molecularweight pure genomic DNA of $1912-2$ strain $\left(A_{260} / A_{280}=2.1\right)$ was prepared according to the Kirby procedure. ${ }^{19}$ Illumina Paired End sequencing and de novo genome assembly was carried out in BaseClear B.V. (Leiden, Netherlands). The genome of 1912-2 consists of 7.124511,0 nucleotides and more than 1000 contigs. The following sequence of an arpA gene was identified with BLAST (National Center of Biotechnological Information, Bethesda, MD, USA; http://www.ncbi.nlm.nih.gov/blast):

5'-ATGGCGAAGCAGGCTCGCGCGGTCCAGACGTGGCGGTCG ATCGTGGATGCCGCGGCGAGCGTCTTCGACGACTACGGCTACG AGCGTGCCGCCATCTCGGAGATTCTCCGCCGCGCCAAGGTC ACCAAGGGGGCCCTGTACTTCCACTTCGCCTCCAAGGAGGCCA TCGCGCAGGCGATCATGGACGAGCAGACCTCCACGGTCGAGT TCGAGCAGGAGGGGTCGCCGCTCCAGTCCCTGGTGGACGGG GGCCAGCAGTTCGCTTTCGCCCTGCGCCACAACGCGATGGCCC GGGCCGGCACCAGGCTCTCCATCGAGGGCGTCTTCCTCGGC GGACCGCATCCGTGGGGCGACTGGATCGACGCGACGGCCCGG ATGCTGGAGCTGGGGCAGGAGCGCGGCGAGGTGTTCCCGCAG ATTGACCCGATGGTGTCGGCCAAAATCATCGTTGCCTCGTTCAC CGGTATCCAGCTGGTCTCGGAGGCCGACACCGGGCGGGCCGA TCTGCGCGAGCAGGTCGCGGAGATGTGGCGCCACATCCTGC CGTCGATCGCTCACCCCGGTGTCATCGCCCACATCAAGCCCGA GGGCCGGGTCGATCTGGCGGCCCAGGCGCGTGAGAAGGCGGA GCGGGAGGAGCAGGAGGCGAGGGTTGCCGCGGAGGCCAAGG GGGCCGGCTCCGATCCCGCGACGGACACGGGCTCCAGGGCCG GCGGGGCGGGCCTGCGGGGCGGTGGATCCCCTCGCGGTGCGC GAGCCGGGCAGGCCGACGACGAGGGTGACGAGGAGCCTGCG GGCGCGGGGGTCGCGGC-3'. 

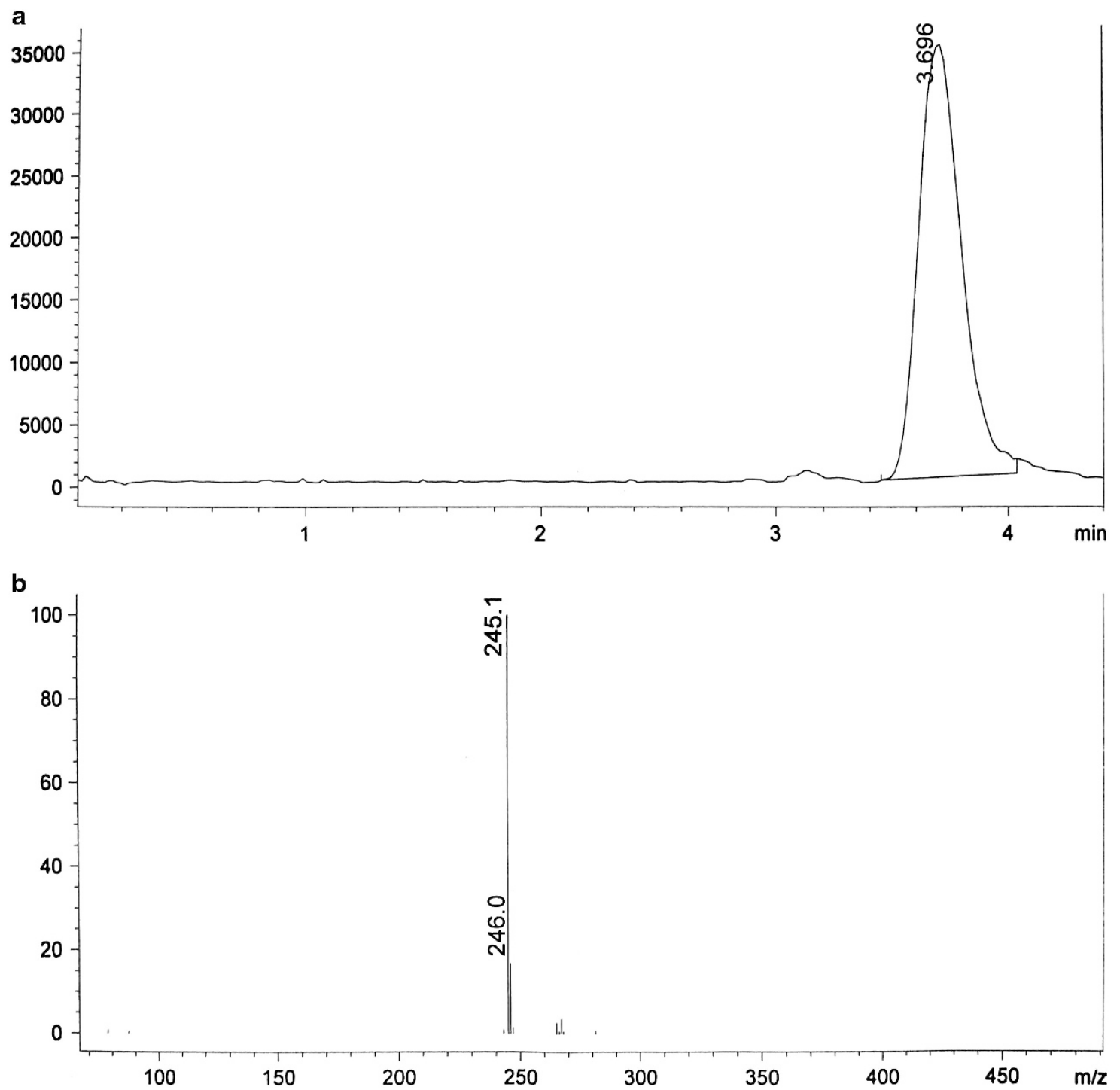

Figure 3 HPLC (a) and HPLC/MS (b) of (L)-N-methylphenylalanyl-dehydrobutyrine diketopiperazine from strain 1912-2.

Table 1 Results of NMR assigments $\left({ }^{1} \mathrm{H},{ }^{13} \mathrm{C}, \mathrm{HSQC}, \mathrm{HMBC}, \mathrm{COSY}\right.$, NOE)

\begin{tabular}{|c|c|c|c|c|c|c|}
\hline & $\begin{array}{c}{ }^{13} \mathrm{C} \\
(125 \mathrm{MHz})\end{array}$ & $\begin{array}{l}\text { Atom } \\
\text { type }\end{array}$ & ${ }^{1} \mathrm{H}(300 \mathrm{MHz})$ & $H M B C$ & $\cos Y$ & NOESY \\
\hline 1 & 165.4 & $\mathrm{C}_{\mathrm{q}}$ & - & & & \\
\hline $1^{\prime}$ & 158.7 & $\mathrm{C}_{\mathrm{q}}$ & - & & & \\
\hline 4 & 134.8 & $\mathrm{C}_{\mathrm{q}}$ & - & & & \\
\hline 5,9 & 129.6 & $2 \mathrm{CH}$ & $7.02(\mathrm{~m})$ & 7 & $6 / 8$ & \\
\hline 6,8 & 128.0 & $2 \mathrm{CH}$ & $7.20(\mathrm{~m})$ & 4 & $5 / 9$ & \\
\hline $2^{\prime}$ & 127.2 & $\mathrm{C}_{\mathrm{q}}$ & - & & & \\
\hline 7 & 126.8 & $\mathrm{CH}$ & $7.20(\mathrm{~m})$ & & & \\
\hline $3^{\prime}$ & 111.6 & $\mathrm{CH}$ & $5.38(q, J)$ & $1^{\prime}$ & & $4^{\prime}$ \\
\hline 2 & 62.7 & $\mathrm{CH}$ & $4.36(\mathrm{~m})$ & $\begin{array}{c}1,1^{\prime}, 3 \\
4\end{array}$ & 3 & \\
\hline 3 & 36.7 & $\mathrm{CH}_{2}$ & $\begin{array}{c}3.15,3.01, A B X, J_{A B}, \\
J_{A X}, J_{B X}\end{array}$ & $\begin{array}{c}1,2,4 \\
5 / 9\end{array}$ & 2 & \\
\hline $\mathrm{NMe}$ & 32.2 & $\mathrm{CH}_{3}$ & $2.97 \mathrm{~s}$ & $1^{\prime}, 2$ & & - \\
\hline \multirow[t]{2}{*}{$4^{\prime}$} & 10.6 & $\mathrm{CH}_{3}$ & $1.41 \mathrm{~d}, \mathrm{~J}$ & $2^{\prime}, 3^{\prime}$ & & $3^{\prime}, \mathrm{NH}$ \\
\hline & & $\mathrm{NH}$ & $9.74 \mathrm{~s} \mathrm{br}$ & & & \\
\hline
\end{tabular}

The sequence of the arpA gene was found in contig 777 (length $=2545$ ), containing 831 nucleotides, 92\% of which have identity with the sequence of the SGR_3731 gene encoding the ArpA protein in S. griseus NBRC 13350. The high identity between the arpA of 1912-2 and SGR_3731 genes explains the biological activity found for the A-factor in the mutant strain S. globisporus 1912-B2. Strain 1912-2 does not produce the $A$-factor. It may contain a mutation in the af $f_{s} A$ gene encoding $A$-factor synthase. Sequencing data gave evidence for such a possibility, because $a f_{s} A$ of $S$. griseus NBRC 13350 and afs $A$ of S. globisporus $1912-2$ are only $83 \%$ identical, significantly less than the corresponding arpA genes (92\% identity). There are eight gaps among 945 nucleotides of $a f_{s} A$ genes and six of them are present in a short 20-nucleotide sequence, which may indicate the localization of mutational changes in this region of the afs $A$ 1912-2 gene:

570-CCG--GCCTCGCCGGCGGG 587 afsA S. griseus NBRC 13350

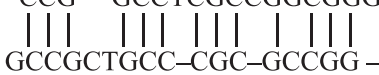

afsA S. globisporus 1912-2

\section{DISCUSSION}

The molecular structure of a new compound that is able to restore the antibiotic biosynthesis and morphogenesis in S. globisporus 1912-B2 


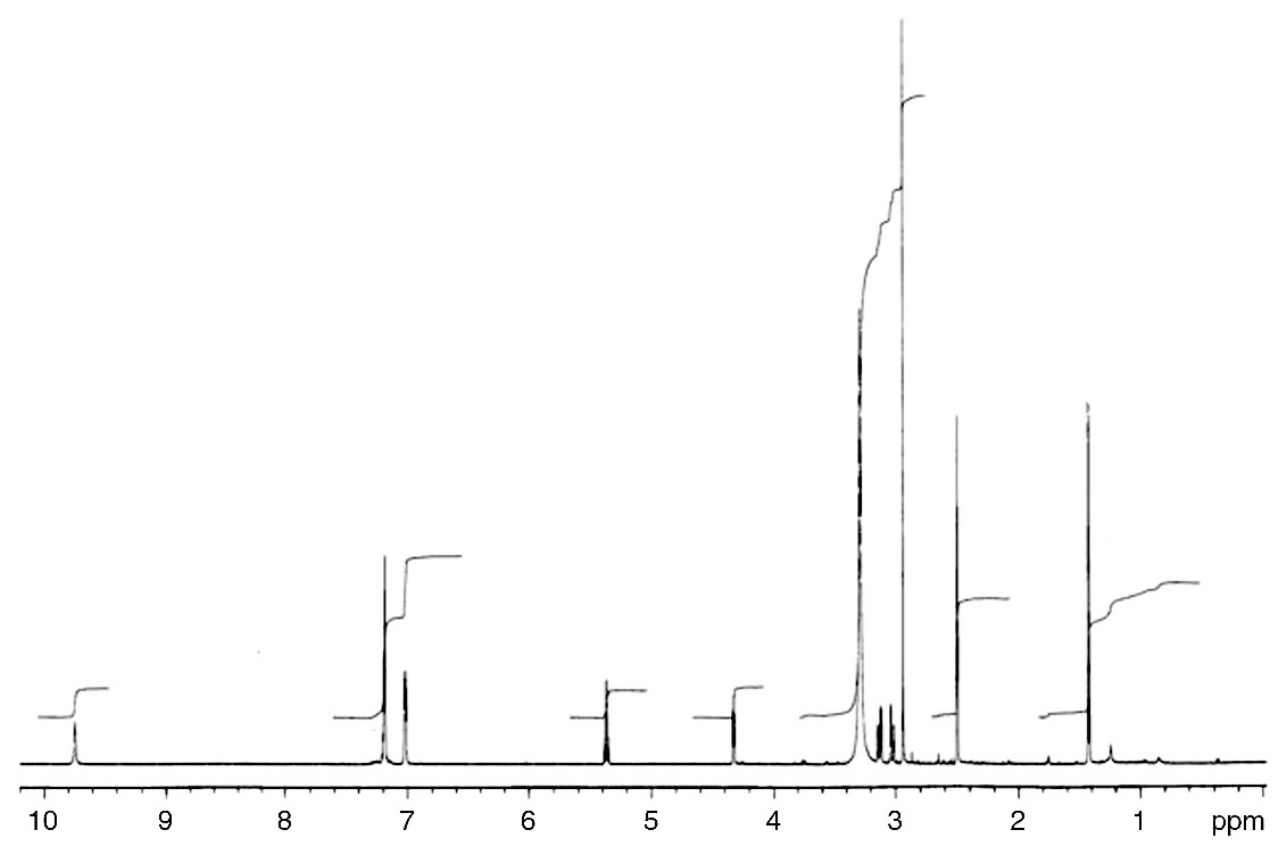

Figure 4 1H NMR spectrum of (L)- $N$-methylphenylalanyl-dehydrobutyrine diketopiperazine from strain 1912-2.
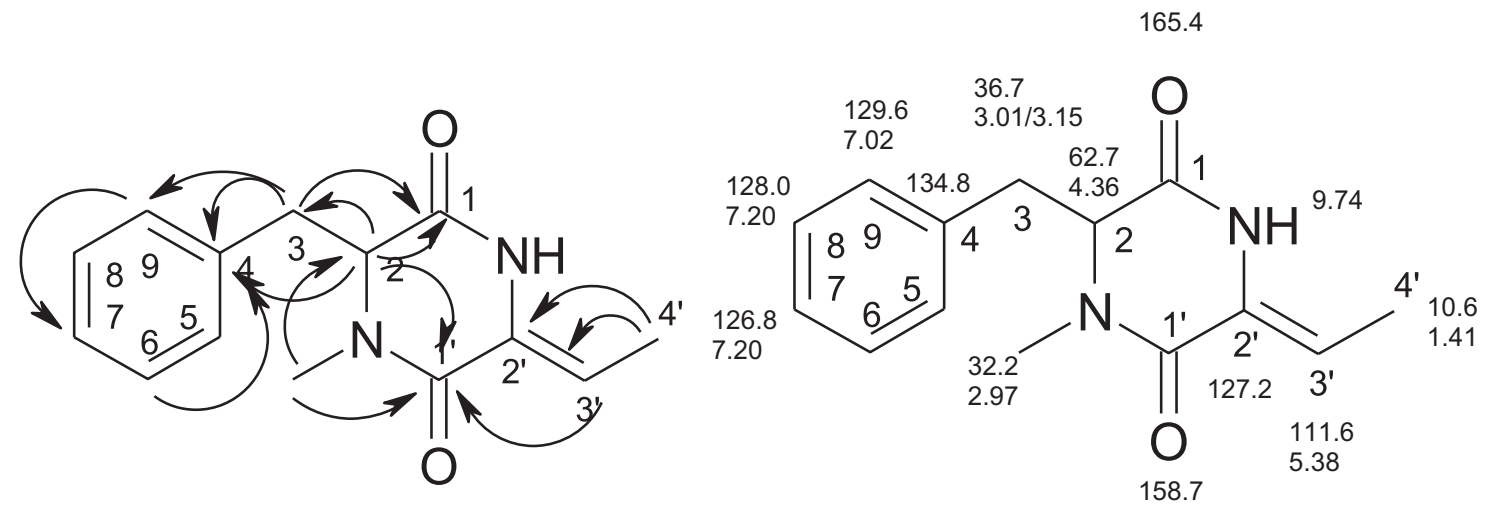

Figure $5 \mathrm{HMBC}$

observed

for L-6-benzyl-3-eth-(Z)-ylidene-1-methyl-piperazine-2,5-dione

$[(\llcorner)-N$-methylphenylalanyl-dehydrobutyrine diketopiperazine).

and S. griseus 1439 has been elucidated as the new DKP MDD. This DKP has not previously been described, although DKPs with similar structures were found as products of streptomycetes and other bacterial species. For instance, L-Phe subunits of other DKPs were paired with L-Pro, ${ }^{20-22}$ L-Ser ${ }^{23}$ or L-Leu. ${ }^{24}$ Dehydrobutyrine was found previously only in a pair with L-Trp. ${ }^{25}$ DKPs are widely produced by microorganisms and exhibit a variety of biological activities, including antibacterial, antifungal, antiviral, anti-prion, antitumor and immunosuppressive. ${ }^{26}$ They also participate in bacterial communication systems as imitators of signalling molecule actions.

The most probable explanation of the biological activity of MDD may be its imitation of the $A$-factor action. According to the literature data, DKPs may mimic the action of $N$-acyl homoserine lactones by interaction with LuxR regulatory proteins and the resulting complex can bind to lux-boxes, specific gene promoter sequencing that affect the expression of quorum sensing target genes. ${ }^{1,4,20}$ ArpA and LuxR proteins belong to the TetR protein superfamily. MDD may interact with the regulatory domain of the receptor protein ArpA in S. griseus 1439 and S. globisporus 1912-B2, imitating $A$-factor action. However, the concentration of MDD must be $\sim 10$ times higher than that of the $A$-factor to achieve the same level of landomycin $\mathrm{E}$ and streptomycin production in the corresponding cultures. A possible reason for this lower activity of MDD may be its lower specificity in comparison with $A$-factor in interaction with receptor protein ArpA.

\section{CONFLICT OF INTEREST}

The authors declare no conflict of interest.

\section{ACKNOWLEDGEMENTS}

This work was supported by the National Academy of Sciences of the Ukraine (0111U000606). 
1 Degrassi, G. et al. Plant growth-promoting Pseudomonas putida WCS358 produces and secretes four cyclic dipeptides: cross-talk with quorum sensing bacterial sensors. Curr. Microbiol. 45, 250-254 (2002).

2 Dunn, A. K. \& Handelsman, J. Toward an understanding of microbial communities through analysis of communication networks. Antonie van Leeuwenhoek 81, 565-574 (2002).

3 Takano, E. $\gamma$-Butyrolactones: Streptomyces signalling molecules regulating antibiotic production and differentiation. Curr. Opin. Microbiol. 9, 287-294 (2006).

4 Ryan, R. P. \& Dow, M. Diffusible signals and interspecies communication in bacteria. Microbiology. 154, 1845-1858 (2008).

5 Sidda, J. \& Corre, C. Gamma-butyrolactone and furan signaling systems in Streptomyces. Methods Enzymol. 517, 71-87 (2012).

6 Ohnishi, Y., Yamazaki, H., Kato, J.-y., Tomono, A. \& Horinouchi, S. AdpA, a central xtranscriptional regulator in the A-factor regulatory cascade that leads to morphological development and secondary metabolism in Streptomyces griseus. Biosci. Biotechnol. Biochem. 69, 431-439 (2005).

7 Horinouchi, S. Mining and polishing of the trove in the bacterial genus Streptomyces. Biosci. Biotechnol. Biochem. 71, 283-299 (2007).

8 Kleiner, E. M. et al. The structure of A-factor, a bioregulator from Streptomyces griseus. Bioorg. Khim. 2, 1142-1147 (1976).

9 Hara, H., Ohnishi, Y. \& Horinouchi, S. DNA microarray analysis of global gene regulation by A-factor in Streptomyces griseus. Microbiology 155, 2107-2210 (2009).

10 Anisova, L. N. et al. Regulators of the development in Streptomyces coelicolor A3(2). Izv. Akad. Nauk. SSSR Biol. 1, 98-107 (1984).

11 Korynevska, A. et al. Mechanisms underlying the anticancer activities of the angucycline landomycin E. Biochem. Pharmacol. 74, 1713-1725 (2007).

12 Ostash, B. et al. Identification and characterization of the Streptomyces globisporus 1912 regulatory gene indYR that affects sporulation and antibiotic production. Microbiology 157, 1240-1249 (2011).

13 Matselyukh, B. P., Tymoshenko, S. G., Bambura, O. I. \& Kopejko, O. P. Screening and characterization of regulators of landomycin E biosynthesis. Mikrobiol. Zh. 73, 16-20 (2011).

14 Matselyukh, B. P., Mohammadipanah, F., Laatsch, H., Rohr, J. \& Efremenkova, O. Purification and structure elucidation of the by-product of new regulator of antibiotic production and differentiation of Streptomyces. Mikrobiol. Z. 74, 66-73 (2012).

15 Borisova, L. N., Ivkina, N. S. \& Rapoport, I. A. Chromatographic study of the mutants of Actinomyces streptomycini induced by nitrosomethylbiuret. Dokl. Akad. Nauk. SSSR 171, 728-732 (1996).

16 Pliner, S. A. et al. Isolation and primary characteristics of A-factor. Bioorg. Chim. 1, 70-76 (1975).

17 Laatsch, H. AntiBase, A Database for Rapid Dereplication and Determination of Microbial Natural Products (Wiley-VCH, Weinheim, Germany. See http://wwwuser.gwdg.de/-hlaatsc/, 2013)

18 Li, W.-R. \& Peng, S.-Z. Rational design and synthesis of unsaturated 2,5-dioxopiperazine derivatives as potential protein tyrosine kinase inhibitors. Tetrahedron Lett. 39 , 7373-7376 (1998).

19 Kieser, T., Bibb, M., Buttner, M., Chater, K. \& Hopwood, D. Practical Streptomyces Genetics (The John Innes Foundation, Norwich, 2000).

20 Holden, M. T. G. et al. Quorum sensing cross talk: isolation and chemical characterization of cyclic dipeptides from Pseudomonas aeruginosa and other Gram-negative bacteria. Mol. Microbiol. 33, 1254-1266 (1999).

$21 \mathrm{Gu}, \mathrm{Q}$., Fu, L., Wang, Y. \& Lin, J. Identification and characterization of extracellular cyclic dipeptides as quorum-sensing signal molecules from Shewanella baltica, the specific spoilage organism of Pseudosciaena crocea during $4^{\circ} \mathrm{C}$ storage. J. Agric. Food Chem. 61, 11645-11652 (2013).

22 Hamza, A. A., Ali, H. A., Clark, B. R., Murphy, C. D. \& Elobied, E. A. Cyclo (L-phenylalanyl, L-prolyl). Diketopiperazines from a newly isolated Streptomyces sudanensis. A4.4.E3. J. Biotechnol. Pharm. Res. 4, 1-7 (2013).

$23 \mathrm{Cho}, \mathrm{J}$. Y. et al. Isolation and structural determination of the antifouling diketopiperazines from marine-derived Streptomyces praecox 291-11. Biosci. Biotechnol. Biochem. 76, 1116-1121 (2012).

24 Lautru, S., Gondry, M., Genet, R. \& Pernodet, J. L. The albonoursin gene cluster of S. noursei biosynthesis of diketopiperazine metabolites independent of nonribosomal peptide synthethases. Chem. Biol. 9, 1355-1364 (2002).

25 Avendano, C., Cabezas, N., de la Cuesta, E. \& Gonzalez, J. F. Reinvestigation of tryptophan-dehydrobutyrine diketopiperazine structure. ARKIVOC ix, 30-38 (2005).

26 Martins, M. \& Carvalho, I. Diketopiperazines: biological activity and synthesis. Tetrahedron 63, 9923-9932 (2007). 